\title{
Asteroid Surveys and other CCD Projects using the Observatoire de la Cote D'Azur (OCA) Schmidt Telescope
}

\author{
Alain Maury \\ Observatoire de la Cote d'Azur - 06460 Caussols - FRANCE \\ Maury@ocar01.obs-azur.fr
}

\begin{abstract}
A wide field CCD camera has been developed for asteroid searches and has been used at our Schmidt telescope since September 1993. This paper describes this camera and its software as well as the changes it introduced in the type of programs that were requested on the telescope, and changes introduced in the way the telescope is operated.
\end{abstract}

\section{Introduction}

Several observatories around the world are currently upgrading their facilities in order to start a new generation of asteroid discovery programs. Since 1989, the Spacewatch group in Tucson (Gehrels 1991) has shown very clearly that CCDs were detecting moving sources, such as asteroids, much more efficiently than photographic plates. This led us to develop a first $2 \mathrm{k} \mathrm{CCD}$ camera and use it on our telescope. The first change produced by this camera is that 80 per cent of the requested observing programs are now CCD-based programs, even though the field of this camera is, so far, only 1 per cent that of a photographic emulsion.

\section{General Facts and Thoughts concerning Asteroids Search Pro- grams}

The distribution of asteroids versus magnitude is an exponential one. Increasing the completeness magnitude of the known sample implies using a technology able to discover several tens of thousands of new objects per year, instead of the few hundreds currently reported annually to the IAU. The incentives for detecting many more fainter asteroids are many :

- Detection of all the potentially dangerous asteroids (we have known for a few years that more than 95 per cent of the asteroids large enough to cause a global catastrophe are still undiscovered (what if ?).

- Providing a better estimate of the current near-Earth object population (and the relationship with the cratering record of inner solar system planets and satellites).

- Discovering possible space mission candidates. 
- Future mining sources for the solar system exploration (long term future).

- Finding many more fly-by candidates in the main belt (Galileo and Cassini type missions ).

- Study the dynamics of the Solar System, asteroid families in the main belt and in the Jupiter Trojan belt.

- Finding new types of objects (outer planets Trojans?).

- Finding a statistically sound sample of the brightest trans-neptunians/ Neptune Trojans objects.

- Discovering more comets of various kinds (short and long periods).

In 1989, the Spacewatch group in Tucson (Arizona) started discovering many faint asteroids using a large CCD ( 0.5 degrees on the sky) on a Newtonian telescope (Gehrels 1991). Only objects with unusual motion are followed up, because of the small field of the detector, and too small angular monthly sky coverage.

\section{The $2 \mathrm{k}$ CCD camera at the OCA Schmidt telescope.}

The fact that Kodak has not been very eager to support astronomical photography in the past ten years (this is an understatement) is also a good reason to start using large CCDs, since this technology now seems ripe for wide field imaging. Eastman Kodak introduced its last emulsion specially designed for astronomical use in the late sixties. As of 1994, only IIIaJ and IIIaF plates are still available. We started using 153-01 plates, and then 4415 films in 1989. Whilst this emulsion is a very interesting detector in panchromatic mode, or in the $\mathrm{R}$ band (even though not easy to hypersensitize), the current choice of Kodak emulsions is not sufficient for regular astrophysical work.

Our first experience with CCDs inside a Schmidt telescope was provided in June 1992, when we built a camera around a Thomson $384 \times 576$ CCD with 23 micron pixels in order to provide users with a better calibration than was achievable using the classical spot sensitometer. This camera was built on a very small budget and ran with a PC 486 computer, used 8200 Exabyte tape drive, and a wire wrapped interface board. Initially it was designed as another plate holder which could be put inside the telescope in less than 5 minutes. Since then, practical considerations related to the wearing of connectors and cables made us change our mind, and now the CCD camera goes inside the telescope for several nights in a row. The controller is a classical JPL/Palomar controller (Gunn et al. 1987). The PC boards for the camera controller were donated by Dr. F. Harris (now at U.S.N.O. Flagstaff, then at Palomar Obs.). The control software (called SPEEDI) was written by a then computer science student, $\mathrm{Mr}$ Savalle, using GNU C++ with a DOS extender so as to bypass the classical PC memory limitations. Shortly afterwards, we tested a Loral $4 \mathrm{k}, 7.5$ micron pixels engineering chip which proved to have a very low dynamic range (less than 1000 $\mathrm{e}^{-}$per pixel). In September 1993, this camera and its software were modified so as to use a Loral 442A CCD chip. The performance of this camera can be summed up as follows: 
- $2048 \times 2048$ square pixels of size 15 micron. One arcsec sampling, 33 arcmin field of view. The chip is a grade 4 low cost $(1994: \$ 2,000)$ commercial CCD (bought at Loral Milpitas). Grade 4 chips are supposed to have up to 60 defective columns at room temperature (most of them however are hot spots). However, at low temperature our chip shows only $4+1$ column parts with defects.

- Field flattening is provided by a single plano-convex lens of focal length $1050 \mathrm{~mm}$ as a camera window. Calculations and sky tests show that this lens does not introduce any measurable aberrations even at one micron wavelength.

- Used in MPP mode, at -35 C temperature, our CCD has a thermal noise of roughly $1 \mathrm{e}^{-}$per second, per pixel. This is achieved by a three stage Peltier cooler and a refrigerated glycol $(-10 \mathrm{C})$ circulation of the warm face. This is the only low cost and compact system that we have been able to find that could operate unattended inside the tight space of a Schmidt telescope plate holder.

- A readout time of $110 \mathrm{~s}$ using a single amplifier. Readout noise is $19 \mathrm{e}^{-}$ (which is nominal, since this chip has a double stage amplifier for high speed readouts).

- Dynamic range is of the order of $120,000 \mathrm{e}^{-}, \mathrm{AD}$ gain of $2.3 \mathrm{e}^{-} / \mathrm{ADU}$.

- Automated 8 position filter wheel, currently fitted with $B, B j, V, R, I$ and $Z 50 \mathrm{~mm}$ square glass filters (Bessell 1990). A very crude qualitative assessment of the performances of this CCD would be to say that the CCD is basically not sensitive to $U$, has a response only slightly better than that of a hypered IIaO plate in the $B$, does much better in the $V$, and has about 10 times the sensitivity of photographic plate in $R$ and $I$, in the sense that a typical 2 to $3 \mathrm{~min} C C D$ exposure goes about as deep as a 20 to $30 \mathrm{~min}$ exposure on hypersensitized IIIaF emulsion. This makes this single detector (which wastes 99 per cent of the light provided by the Schmidt telescope) only a factor of ten worse than photographic plates in this passband. One can of course question whether a CCD can achieve better faint detections than that of a correctly processed and amplified fine grained emulsion. While the sensitivity in $Z$ band ( 1 micron) is not terrific, it does perform several orders of magnitudes better than the $I-Z$ emulsion, and some interesting results have already been obtained on full moon nights in this band.

- Flat fields can be obtained with a light box of special design mounted on the telescope dome or done during the twilight period.

- A typical exposure time is $3 \mathrm{~min}$, and a new image can be acquired every 5 min. A good 8 hours night will allow us to acquire more than 500 Mbytes of data.

A Hunt technology mother board for a Texas Instruments 320C40 Digital Signal Processor (DSP) TIM board has been recently added to this system so 
as to provide an intelligent interface to the camera. This TIM board features a C40 with RAM (1 Mbyte), 6 parallel ports for interface with the external world, and a 50 Mflops CPU. An interrupt driven software has been written by L. Barbano (OCA) and allows us to take data from the camera and send it to the PC and use the camera in scan mode. It will also soon allow to us to perform data pre-reduction (flat fielding) and part of the automatic detection software. A new 5 Gbytes 8505 Exabyte tape drive has replaced the older 8200 unit. A direct DSP to Exabyte SCSI interface has been contracted to the French firm AITA.

The observing room of the telescope has been modified to take into account the new system. Apart from the Macintosh which already controlled the telescope, and the PC which controls the CCD camera, a new HP9000-710 workstation has been purchased so as to do the data reduction. This station has 64 Mbytes of RAM, 2 Gbytes of hard disk space and a 12 Mflops CPU. It runs a home made software for automated detection, but we use IRAF for "regular" data reduction. All three computers are linked to the Internet, and have names which are both Earth Grazing Asteroids and Gaulish Gods (Toutatis, Epona and Taranis.obs-azur.fr).

It is possible to compare the use of this CCD system with the regular photographic mode from an observer's point of view :

- The CCD observing is much more tiring than regular photographic observing since the operations requires constant attention because of the very short exposure times. This is not a trivial matter, since the night is also longer because of twilight flatfields. It is clear to us that it is necessary to automate the telescope and camera operation so as to be able to optimize the use of the telescope (i.e. computers do not fall asleep at 4 a.m). After studying the Berkeley Automated Imaging Telescope software (Richmond 1993), we are currently writing a software dubbed CATS (Controle Automatique du Telescope de Schmidt) which will fully automate the use of the telescope. To do this we defined several rules which allows the software to define priorities among the fields to be taken. Apart from a few detailed points, our observing request files are compatible with those of the Berkeley group. A WWW interface will soon be written in order to allow registered users to request CCD observations.

- An $f / 2.5$ or $f / 3.5$ relative aperture provides a large numbers of photons per unit time, and except for very small exposures times or exposures with narrow band filters, the data is very quickly and strongly photon noise limited. A simple model of the variation of noise versus readout noise shows clearly that a CCD controller for a Schmidt telescope should provide a very fast readout time compared to normal CCD controllers. Provided that the readout noise increases with the inverse square root of the readout time, it can be shown that an integration of 2 min followed by a "normal" 2 min readout time (in our case with a $19 \mathrm{e}^{-}$readout noise) will typically have 40 per cent smaller sky signal-to-noise ratio than an integration time of $3 \mathrm{~m} 40 \mathrm{~s}$ (almost twice the integration time) followed by a short $20 \mathrm{~s}$ (readout noise up to $40 \mathrm{e}^{-}$) readout sequence. 
- The current telescope shutter, which takes about $3.5 \mathrm{~s}$ to open or close, is not fast enough to image most reference stars. A new curtain-type one, using pneumatic pistons as actuators, and providing $0.1 \mathrm{~s}$ opening time, also able to shutter the future array is currently being built.

Even before the system went through final tests, several programs had already requested the use of this new facility. These programs deal for example with the detection of geostationary satellites; the detection of faint giant planets satellites; multi-colour photometry of stars; astrometry for parallaxes in selected fields; photometry of large globular clusters, ensemble photometry in open clusters; photometry of large galaxies and large Abell clusters; detection of gravitational lenses in the central region of M31; detection of quasar candidates through multi-colour photometry (see Moreau in this volume).

This has obliged us to consider more seriously the problem of data distribution to our users, and we should purchase a DAT tape so as to offer three types of digital media : network for programs consisting of a small number of images (using our anonymous ftp account at rossini.obs-nice.fr), DAT and Exabyte otherwise. Ideally, we will strive to provide only archiveable data, which means pre-processed, recentered, cleaned and stacked images to users. Because of lack of time and money, no decisions have been taken yet as far as data archiving is concerned.

\section{Automated asteroid detection software}

The general idea behind automated asteroid detection is to observe the same field at different times during a given night, pre-process them in a normal fashion (bias, flat field correction and cosmic ray removal), then run an automated detection software which converts the images into lists of detected objects. After the calculation of the relative offsets between these frames, the "static" objects (i.e. stars and galaxies) are removed, and objects having a linear motion during the night are matched together. The last step is then to convert $x, y$ values and fluxes into right ascensions, declinations and magnitudes using the NASA Guide Star Catalog. There were two points to take into account while developing our software :

(i) We are currently building a multi-CCD camera ( 9 chips). When it comes into operation, we will have to rely solely on the computer to detect sources, i.e. we will not have the manpower to confirm visually all the sources detected in a single night (typically 15 Gbytes).

(ii) Because it is not possible to follow up correctly the very fast moving objects, we will not worry about detecting trailed asteroids. This question will only be dealt with if we get a very large computer. Hence our software provides only information on the positions and fluxes of the detected sources.

R. Savalle worked in collaboration with the image processing group at the OCANice (A. Bijaoui) to create a relatively original and efficient software based on adaptive filtering. Mostly because of the computer network used (see above), 
it has been dubbed GODS (for Global Orbit Determination System). It is a client-server application which uses a convolution by a wavelet, the size of which is matched to the seeing conditions. A single loop allows one to perform this convolution as well as thresholding, maxima detection and characterisation of the detected sources. The second step (matching) is performed after the catalogue of detected sources has been rearranged in Voronoi trees, which provide very efficient searches through these catalogues. To do this we used a package called LEDA. The reduction procedure has been written by G. Hahn (DLR Germany). Tests have shown problems with bloomed stars and so we have made modifications and now operate in a partially reversed mode using dynamic antiblooming (Neely \& Janesick 1993). The current version of this software processes a full $2 \mathrm{k}$ frame (typically 2500 detected sources) in about $7 \mathrm{~min}$ on our Unix workstation. It is being ported to the 320 C40 DSP where we expect to achieve real time performance.

\section{References}

Bessell M.S., 1990, PASP, 102, 1181

Gehrels T., 1991, In Scanning with CCDs, Space Sci. Rev. 58, 347-375

Gunn J. E., Emory E.B., Harris F. H. \& Oke J.B., 1987, PASP, 99, 518

Neely S. \& Janesick J., 1993, PASP, 105, 1330

Richmond M. W., Treffers R. R. \& Filippenko A.V., 1993, PASP, 105, 1164 